\title{
Internal and external validity: can you apply research study results to your patients?
}

\author{
Cecilia Maria Patino 1,2,a, Juliana Carvalho Ferreira ${ }^{1,3, b}$
}

\section{CLINICAL SCENARIO}

In a multicenter study in France, investigators conducted a randomized controlled trial to test the effect of prone vs. supine positioning ventilation on mortality among patients with early, severe ARDS. They showed that prolonged prone-positioning ventilation decreased 28-day mortality [hazard ratio $(\mathrm{HR})=0.39 ; 95 \% \mathrm{CI}: 0.25-0.63$. $^{(1)}$

\section{STUDY VALIDITY}

The validity of a research study refers to how well the results among the study participants represent true findings among similar individuals outside the study. This concept of validity applies to all types of clinical studies, including those about prevalence, associations, interventions, and diagnosis. The validity of a research study includes two domains: internal and external validity.

Internal validity is defined as the extent to which the observed results represent the truth in the population we are studying and, thus, are not due to methodological errors. In our example, if the authors can support that the study has internal validity, they can conclude that prone positioning reduces mortality among patients with severe ARDS. The internal validity of a study can be threatened by many factors, including errors in measurement or in the selection of participants in the study, and researchers should think about and avoid these errors.

Once the internal validity of the study is established, the researcher can proceed to make a judgment regarding its external validity by asking whether the study results apply to similar patients in a different setting or not (Figure 1). In the example, we would want to evaluate if the results of the clinical trial apply to ARDS patients in other ICUs. If the patients have early, severe ARDS, probably yes, but the study results may not apply to patients with mild ARDS. External validity refers to the extent to which the results of a study are generalizable to patients in our daily practice, especially for the population that the sample is thought to represent.

Lack of internal validity implies that the results of the study deviate from the truth, and, therefore, we cannot draw any conclusions; hence, if the results of a trial are not internally valid, external validity is irrelevant. (2) Lack of external validity implies that the results of the trial may not apply to patients who differ from the study population and, consequently, could lead to low adoption of the treatment tested in the trial by other clinicians.

\section{INCREASING VALIDITY OF RESEARCH STUDIES}

To increase internal validity, investigators should ensure careful study planning and adequate quality control and implementation strategies-including adequate recruitment strategies, data collection, data analysis, and sample size. External validity can be increased by using broad inclusion criteria that result in a study population that more closely resembles real-life patients, and, in the case of clinical trials, by choosing interventions that are feasible to apply. ${ }^{(2)}$

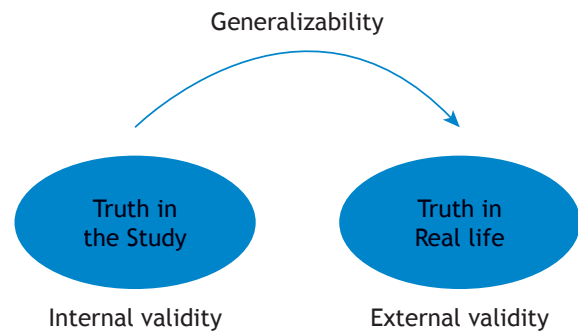

Figure 1. Internal and external validity.

\section{REFERENCES}

1. Guérin C, Reignier J, Richard JC, Beuret P, Gacouin A, Boulain T, et al. Prone positioning in severe acute respiratory distress syndrome. N Engl J Med. 2013;368(23):2159-68. https://doi.org/10.1056/NEJMoa1214103

2. Akobeng AK. Assessing the validity of clinical trials. $J$ Pediatr Gastroenterol Nutr. 2008;47(3):277-82. https://doi.org/10.1097/ MPG.0b013e31816c749f 